\title{
Comparison of sonographic characteristics of primary thyroid lymphoma and anaplastic thyroid carcinoma
}

\author{
Li-Shuang Gu ${ }^{1 *}$, Ning-Yi Cui ${ }^{1 *}$, Yong Wang ${ }^{1}$, Shu-Nan Che ${ }^{2}$, Shuang-Mei Zou ${ }^{3}$, Wen $\mathrm{He}^{4}$, Jun-Ying \\ Liu $^{5,6}$, Xuan-Tong Gong ${ }^{7}$
}

${ }^{1}$ Department of Ultrasound, ${ }^{2}$ Department of Diagnostic Imaging, ${ }^{3}$ Department of Pathology, National Cancer Center/Cancer Hospital, Chinese Academy of Medical Sciences and Peking Union Medical College, Beijing 100021, China; ${ }^{4}$ Department of Ultrasound, Beijing Tiantan Hospital, Capital Medical University, Beijing 100050, China; ${ }^{5}$ Department of Ultrasound, Fudan University Shanghai Cancer Center, Shanghai 200032, China; ${ }^{6}$ Department of Oncology, Shanghai Medical College, Fudan University, Shanghai 200032, China; ${ }^{7}$ Department of Ultrasound, Fatou Community Health Center, Beijing 100023, China

Contributions: (I) Conception and design: Y Wang, NY Cui, LS Gu, W He; (II) Administrative support: Y Wang, NY Cui; (III) Provision of study materials or patients: NY Cui, Y Wang, SM Zou, JY Liu; (IV) Collection and assembly of data: LS Gu, NY Cui, Y Wang, SN Che, XT Gong; (V) Data analysis and interpretation: LS Gu, NY Cui, Y Wang; (VI) Manuscript writing: All authors; (VII) Final approval of manuscript: All authors. *These authors contributed equally to this work.

Correspondence to: Yong Wang. Department of Ultrasound, National Cancer Center/Cancer Hospital, Chinese Academy of Medical Sciences and Peking Union Medical College, Panjiayuannanli No.17, Chaoyang District, Beijing 100021, China. Email: drwangyong77@163.com; Wen He. Department of Ultrasound, Beijing Tiantan Hospital, Capital Medical University, No. 6 Tiantan Xili, Dongcheng District, Beijing 100050, China. Email: ttyyus@sina.com.

Background: Although primary thyroid lymphoma (PTL) and anaplastic thyroid carcinoma (ATC) both account for a rare portion of the morbidity of all thyroid malignancies, the therapeutic methods and prognosis for these two diseases are different. The purpose of this study was to investigate the sonographic characteristics of PTL and ATC and to compare the sonographic findings of PTL and ATC.

Methods: The study included 42 patients with histopathologically proven PTL ( $\mathrm{n}=27)$ and ATC ( $\mathrm{n}=15)$. The Clinical characteristics and sonographic findings were retrospectively reviewed and compared between the two groups.

Results: The mean age of patients with ATC was not significantly different from that in patients with PTL $(\mathrm{P}=0.601)$. The female-to-male ratio of patients with ATC was significantly lower than that of patients with PTL ( $\mathrm{P}=0.029)$. Both PTL and ATC commonly present as a relatively large, solid mass on sonography with compressive symptoms, in which hoarseness was seen more frequently in ATC group (66.7\%) than in PTL group (14.8\%) ( $\mathrm{P}=0.001)$. There is no significant difference in thyroid size, nodular size, margin, shape, echo texture, echogenicity, cystic change, vascularity and local invasion on sonography between ATC and PTL groups. Echogenic strands, markedly hypoechoic and enhanced posterior echo were seen more frequently in PTL group $(92.6 \%, 92.6 \%$, and 85.2\%, respectively) than those in ATC group (6.7\%, 60.0\%, and 33.3\%, respectively) $(\mathrm{P}<0.05)$, and calcification was seen more frequently in ATC group $(80.0 \%)$ than in PTL group (0\%) $(\mathrm{P}<0.001)$. Three ultrasound patterns were observed for PTL including diffuse type (25.9\%), nodular type $(48.2 \%)$ and mixed type (25.9\%), while all ATC cases presented with nodular type (100.0\%). Associated Hashimoto's thyroiditis occurred more frequently in PTL group (59.3\%) than in ATC group (20.0\%) (P=0.023). Conclusions: Certain sonographic features as a markedly hypoechogenicity, the presence of an enhanced posterior echo and linear echogenic strands, lack of calcification and associated Hashimoto's thyroiditis were valuable for distinguishing PTL from ATC. In contrast, heterogeneous echogenicity, uncircumscribed margin, irregular shape, and vascular pattern were not specific features for differential diagnosis.

Keywords: Thyroid carcinoma; anaplastic; lymphoma; ultrasonography

Submitted Aug 05, 2017. Accepted for publication Sep 07, 2017.

doi: $10.21037 /$ jtd.2017.09.48

View this article at: http://dx.doi.org/10.21037/jtd.2017.09.48 


\section{Introduction}

Anaplastic thyroid carcinoma (ATC) is a rare disease, representing approximately $1.3 \%$ to $9.8 \%$ of all thyroid malignancies globally (1). It is the most lethal form of thyroid malignancies, with a high rate of regional and distant spread and a grave prognosis (2-4). Primary thyroid lymphoma (PTL) is also an uncommon disease, representing less than $5 \%$ of thyroid malignancies. PTL is a kind of potentially curable thyroid malignancy. The therapeutic methods for these two diseases are different. Therapeutic strategies for ATC include local therapy alone (surgery or radiotherapy or surgery plus radiotherapy) or combined multimodality treatment $(5,6)$. As for patients with PTL, multiply periodic chemotherapy is the main treatment. The two diseases both present as a rapidly enlarging cervical mass with similar symptoms $(7,8)$. It is clinically valuable to establish an accurate diagnosis promptly, in order to provide appropriate therapy. To our knowledge, only single case reports or small case series have been published and there have been no previous study comparing the sonographic findings between PTL and ATC. Therefore, the aim of this study was to investigate the sonographic characteristics of ATC and PTL and to compare the sonographic findings of ATC and PTL.

\section{Methods}

\section{Patient selection}

From a retrospective review of the pathologic database at our institute between November 1999 and March 2017, 108 patients with PTL and 48 patients with ATCs were included in the present study. Eighty-one patients with PTL and 33 patients with ATC with incomplete imaging data were excluded. Finally, 27 patients with PTL and 15 patients with ATC were included in this study. Thirty-six patients underwent surgery. We retrospectively reviewed the clinical characteristic and sonographic features of these patients. The study was approved by the Hospital Ethics Committee. Each patient was consent informed.

\section{Imaging technique and interpretation}

Thyroid sonography was performed by using one of three scanners (GE logic E9, Philips IU 22, Siemens Acuson S2000) with a 5-12 MHz linear transducer and a 3-5 MHz curve transducer. Ultrasonography analysis was determined separately by two radiologists (with more than
20 and 7 years of experiences in thyroid imaging diagnosis, respectively) without any previous knowledge of the pathologic results. If they have the different viewpoints, characteristics and diagnosis of sonograms would be discussed in consensus.

The following imaging characteristics were reviewed and documented: The size of thyroid, nodule size, margin, shape, echogenicity, echotexture, calcification, cystic change, echogenic strands, posterior acoustic enhancement and vascularity. The sonographic patterns of lesions were divided into three types according to Ota (9): diffuse type, nodular type and mixed type. Nodular type is confined to a unilateral lobe, and the boundary between the tumor and surrounding tissue is clear. Diffuse-type involves two lobes, and the lesion has no clear boundary. Mixed-type is characterized by multiple patchy lesions with hypoechoic regions in the thyroid. The nodule size was recorded as the greatest diameter of each nodule. According to standard of Xia (10), goiter was determined as the presentation of a thyroid with the craniocaudal diameter more than $6.0 \mathrm{~cm}$ or the anteroposterior diameter more than $2.0 \mathrm{~cm}$. The margin was classified as being either circumscribed or uncircumscribed, and the shape was categorized as being regular (oval to round) or irregular. Echo texture was classified as homogeneous or heterogeneous. Internal echogenicity was classified as hypoechoic or markedly hypoechoic. Marked hypoechogenicity was defined as decreased echogenicity compared with the surrounding strap muscle. The presence or absence of calcification, cystic changes and echogenic strands was evaluated. The vascularity was classified as absence or not rich vascularity or rich vascularity.

\section{Pathologic evaluation}

All of the pathologic specimens were reviewed by the pathologist with plentiful experience in thyroid pathology. In this study, all the diagnosis of pathology was according to the 2008 WHO new classification standard for diagnosis and classification.

\section{Statistical analysis}

Clinical and sonographic characteristics were compared between PTL and ATC by the Mann-Whitney test for quantitative parameters and the $\chi 2$ test or Fisher exact test for qualitative parameters. Statistical analyses were performed with SPSS version 20.0 software (IBM 
Table 1 Comparison of clinical characteristics between patients with PTL and ATC

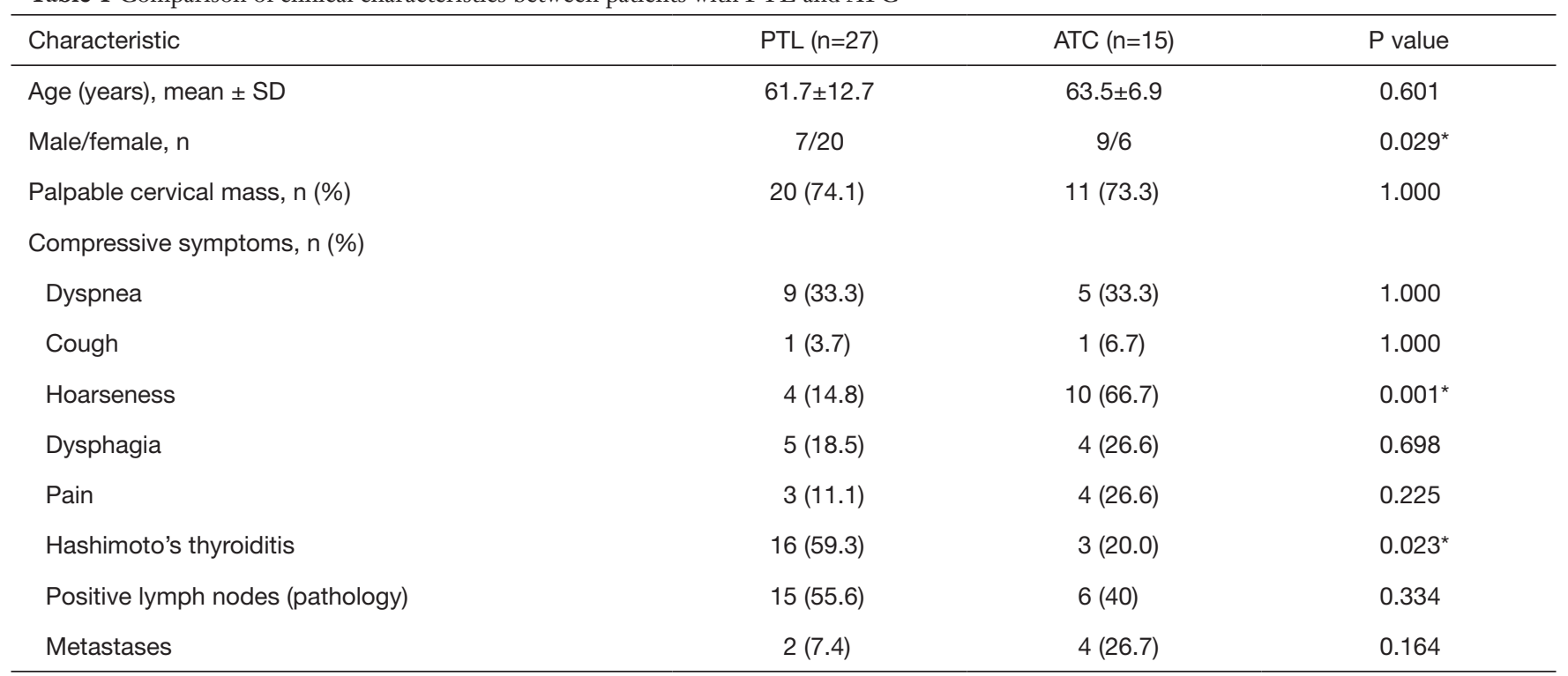

*, $\mathrm{P}<0.05$, difference was statistically significant. ATC, anaplastic thyroid carcinoma; PTL, primary thyroid lymphoma.

Corporation, Armonk, NY, USA), and a $\mathrm{P}<0.05$ was considered statistically significant.

\section{Results}

The clinical and sonographic characteristics are summarized in Tables 1 and 2. Among the 42 patients included in the study, the mean age of patients in the ATC group was not significantly different from that in patients with PTL $(\mathrm{P}=0.601)$. The female-to-male ratio was lower than that of patients with PTL $(\mathrm{P}=0.029)$. The disease courses of ATC and PTL were between 7 days to 30 years (median time 11 months). The patients with PTL had a median course of 18 months (from 10 days to 30 years), and that with ATC had a median course of 11 months (from 1 month to 20 years). Twenty (74.1\%) patients with PTL and $11(73.3 \%)$ patients with ATC have the symptom of palpable cervical masses. Hoarseness was seen more frequently in ATC group (66.7\%) than in PTL group $(14.8 \%)(\mathrm{P}=0.001)$. There are no significant difference in other neck compression symptoms including dyspnea, dysphagia or cough $(\mathrm{P}>0.05)$. Associated Hashimoto's thyroiditis occurred more frequently in PTL group (59.3\%) than in ATC group (20.0\%) ( $\mathrm{P}=0.023)$.

The dominant sonographic findings of PTL were large (average $4.8 \pm 1.7 \mathrm{~cm}$, range $1.6-8.7 \mathrm{~cm}$ ), solid $(100 \%)$, ill-defined $(81.5 \%)$ mass with echogenic strands
(92.6\%), posterior echo enhancement $(85.2 \%)$ and without calcification (100\%) (Table 2), while the dominant sonographic findings of ATC were large (average $5.8 \pm 2.1 \mathrm{~cm}$, range $2.5-11.0 \mathrm{~cm})$, solid $(100 \%)$, ill-defined $(100 \%)$ mass with macrocalcification or microcalcification (80\%) (Table 2). PTL cases presented with diffuse type (25.9\%) (Figure 1), nodular type (48.2\%) (Figure 2) and mixed type (25.9\%) (Figure 3). All ATC cases presented with nodular type (100\%) (Figures 4,5). There is no significant difference in thyroid size, nodular size, margin, shape, echo texture, cystic change and vascularity on sonography between ATC and PTL groups (Table 2).

With regard to tumor-spreading patterns, in 15 patients with ATC, 13 patients had extra-thyroid extension into the surrounding soft tissue (Figure 4A), 1 patients invaded trachea, and 2 patients invaded jugular vein. In 27 patients with PTL, 14 patients had extra-thyroid extension into the surrounding soft tissue (Figures $2 A, B, 3 B$ ), 1 patients invaded trachea (Figure 1C).

In 15 patients with ATC, metastatic tumors were found in the lung $(\mathrm{n}=3)$ and mediastinum $(\mathrm{n}=1)$. In 27 patients with PTL, metastatic tumors were found in the lung $(n=1)$ and axillary lymph nodes $(\mathrm{n}=1)$. There is no significant difference in metastatic lymphadenopathy $(\mathrm{P}=0.334)$ and distant metastasis between two groups $(\mathrm{P}=0.164)$.

Diffuse large B-cell lymphoma (DLBCL) was histopathologically diagnosed in 15 patients; mucosa- 
Table 2 Comparison of sonographic characteristics between patients with PTL and ATC

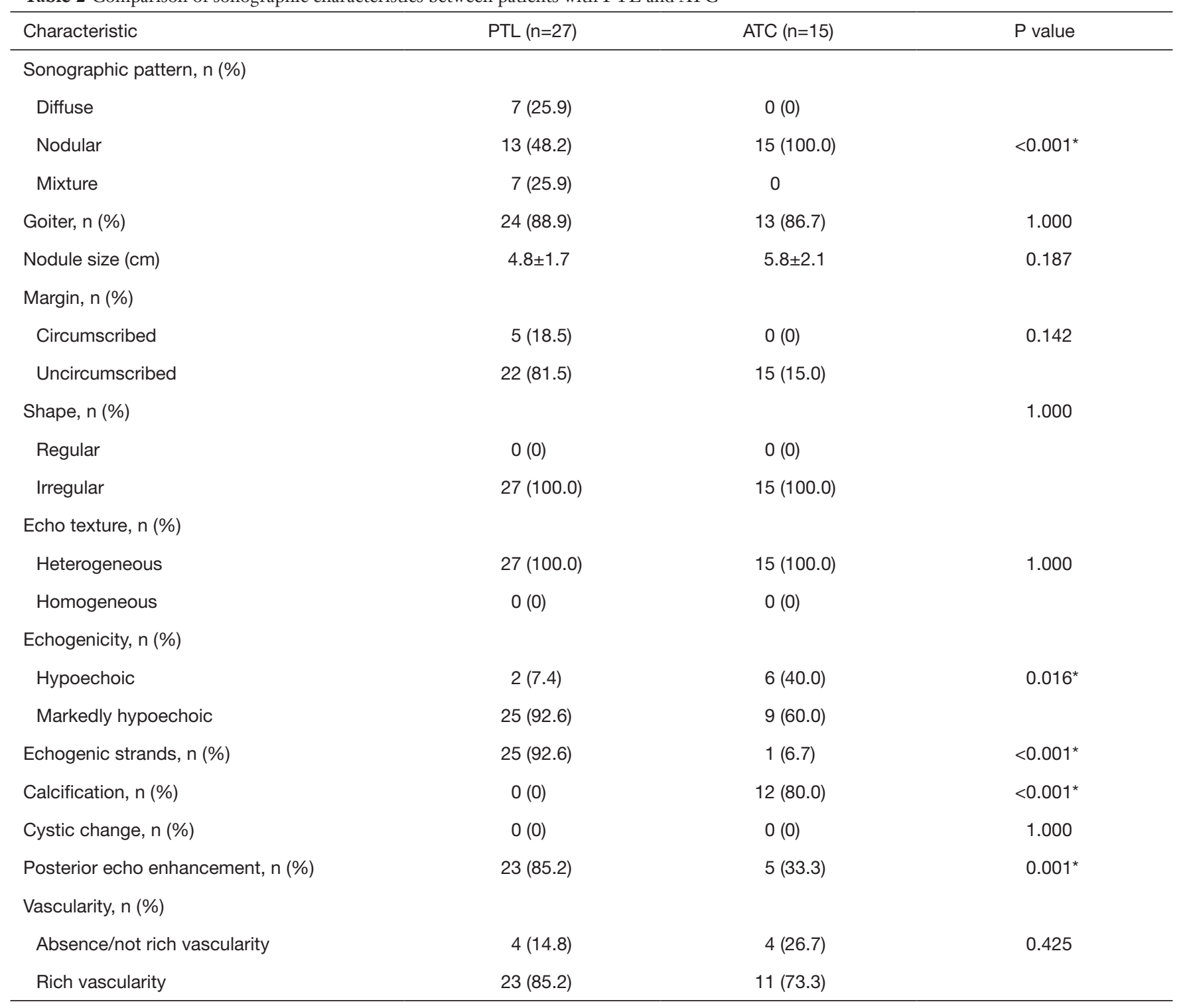

${ }^{*}, \mathrm{P}<0.05$, difference was statistically significant.

associated lymphoid tissue (MALT) lymphoma in 8; follicular lymphoma (FL) in 1; and MALT lymphoma coexisting with DLBCL in 2, FL coexisting with DLBCL in 1.

\section{Discussion}

ATC and PTL are both rare thyroid malignancies. It is important to differentiate PTL from ATC as these disease entities carry a different prognosis and have a different treatment strategy. ATC has extremely poor prognosis with a mean survival of 6 months $(11,12)$, while PTL has generally good prognosis if treated appropriately, with an overall 5 -year survival ranging between $50 \%$ and $60 \%$ (13). Hence, early preoperative diagnosis is important to help make the treatment protocol and improve prognosis.

Ultrasonography has been proven to be the most efficient first-line method for the diagnosis of thyroid diseases with advantages of non-invasiveness, real-time scanning, easy performance, and wide availability. Meanwhile, it is also a mean of assessing the degree of involvement of surrounding soft tissues as well as the area involved $(9,14-16)$. Although CT or MRI is more useful in evaluating larger masses of 

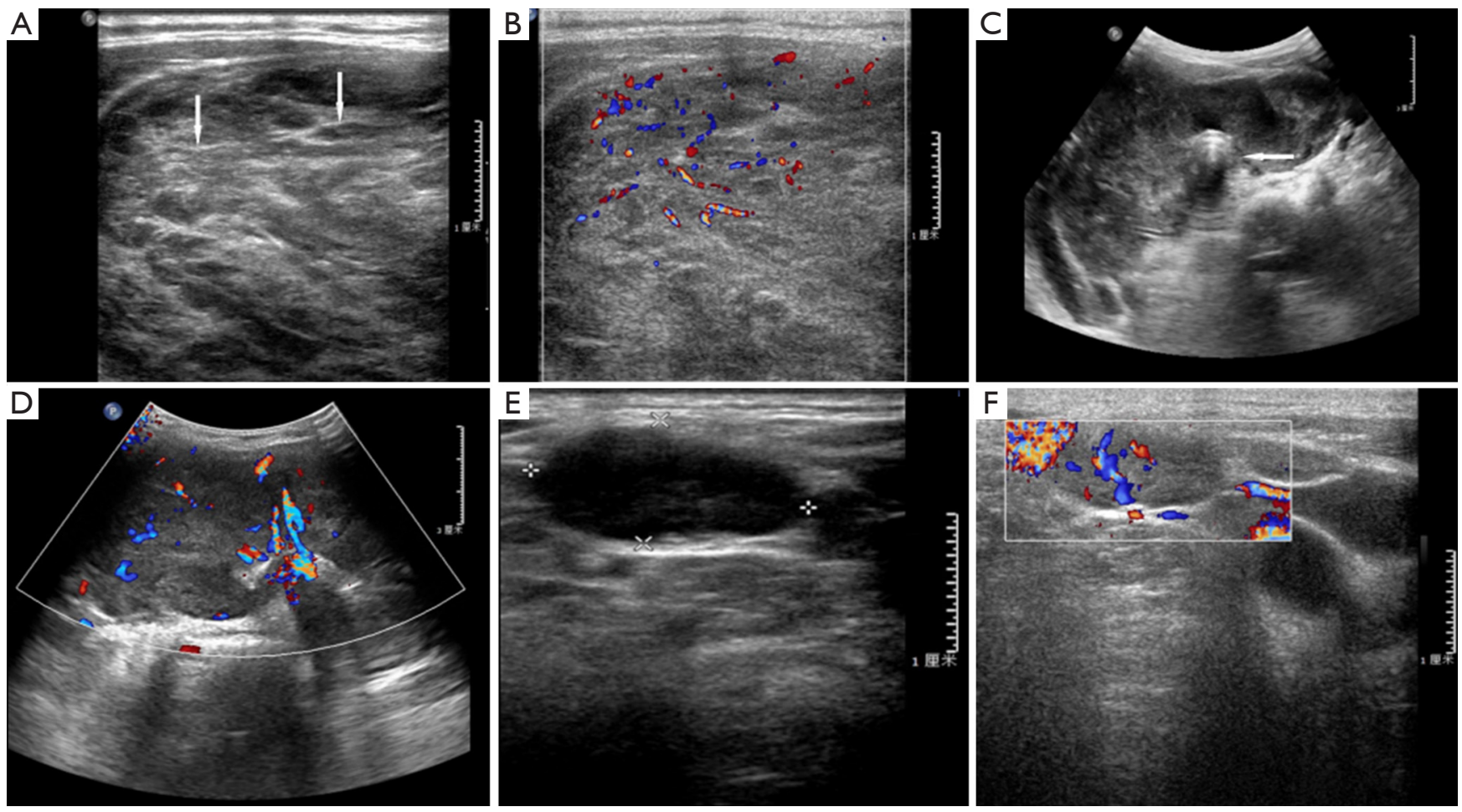

Figure 1 Ultrasonography of MALT lymphoma of the thyroid in a 68-year-old woman presented with gradually growing neck swelling for 3 years. (A) Transverse image of ultrasonography (with linear transducer) of the right thyroid gland showed a diffuse type of lesion with intervening linear echogenic structures (arrows); (B) Color Doppler imaging (with linear transducer) showed a rich internal blood flow; (C) transverse image of ultrasonography (with curve transducer) showed asymmetric diffuse enlargement of the thyroid, replacing the entire two lobes, with invasion of trachea (arrow); (D) Color Doppler imaging (with curve transducer) also showed a rich internal blood flow pattern; (E) longitudinal image of ultrasonography (with linear transducer) showed pathologically confirmed involved lymph nodes in the right cervical IV region appeared as markedly hypoechoic lymph nodes lacking echogenic hilum; (F) Color Doppler imaging of the lymph node showed a rich internal clutter distributed blood flow pattern. MALT, mucosa-associated lymphoid tissue.

thyroid, and is important to allow accurate staging with some functional MRI techniques (such as IMRT) (17).

When ATC or PTL is clinically suspected, US-guided fine-needle aspiration (FNA) or core needle biopsy is initially performed. The diagnostic sensitivity of FNA for the diagnosis of ATC or PTL reported in the literature has varies widely, from $25 \%$ to $90 \%(13,15,18-24)$. Usually, for PTL and ATC, core needle biopsy may become necessary when ATC or PTL is suspected in order to obtain enough tissue.

We retrospectively analyzed clinical and sonographic characteristics of 42 patients (27 PTL and 15 ATC). In our study, PTL and ATC are more common in the elderly (mean age $61.7 \pm 12.7,63.5 \pm 6.9$ years, respectively) without significant differences in age between two groups. PTL has been found more common in female $(20 / 27,74.1 \%)$ whereas
ATC more common in male (9/15, 60.0\%). Previous research showed that both PTL and ATC are usually found in the older female patients (8). From the result of $\mathrm{Ha}$ (24), both ATC and PTL are more common in elderly women, with no significant differences in age and sex. This discrepancy may be because of fewer patients in our ATC group.

PTL and ATC both presented with recently growing thyroid mass and compressive symptoms $(1,25)$. It is often difficult to distinguish between clinical symptoms of PTL and ATC. In our study, 20 of 27 (74.1\%) PTL and 11 of $15(73.3 \%)$ ATC have palpable cervical mass. Seventeen (40.5\%) patients had the fast-growing neck masses in a short time ( $<1$ month). Most patients with PTL or ATC had compressive symptoms such as dyspnea, dysphagia, cough and hoarseness. Among them, hoarseness is more frequent in ATC $(10 / 15,66.7 \%)$ than PTL $(4 / 27,14.8 \%)(\mathrm{P}=0.001)$, 

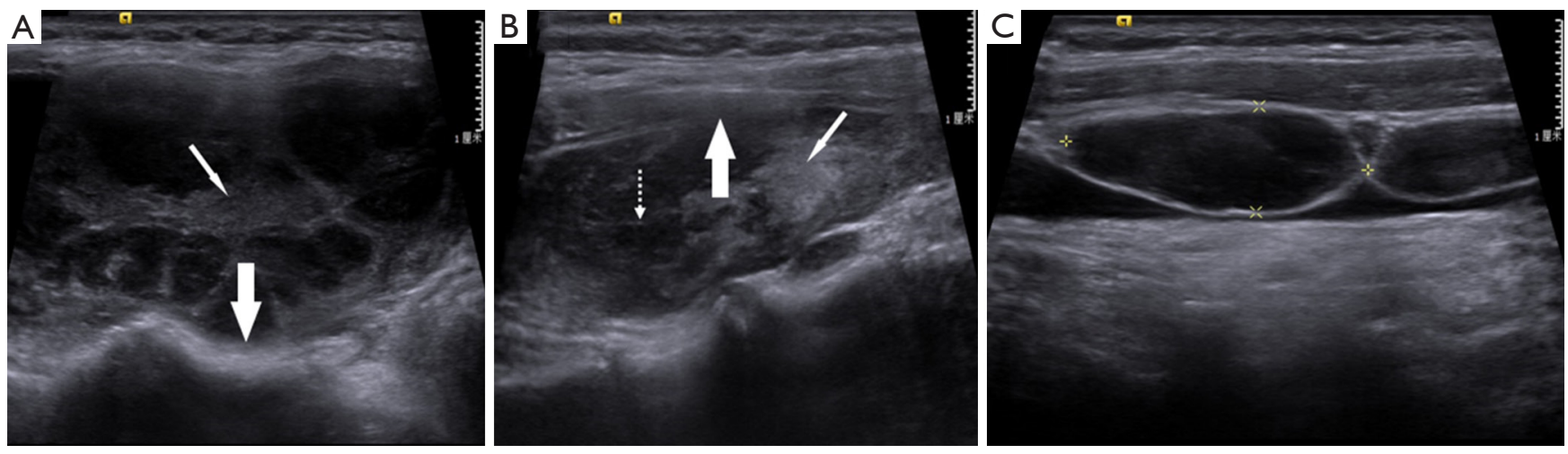

Figure 2 Ultrasonography of MALT lymphoma coexisting with DLBCL of the thyroid in a 48-year-old woman presented with a neck mass for 1 year. (A) Longitudinal image of ultrasonography (with linear transducer) showed mixed type of PTL. Multiple markedly hypoechoic segments were patchily present in the right lobe of the thyroid, and normal thyroid tissues can be seen between the segments (thin arrow). The capsule of the thyroid was invaded (thick arrow); (B) longitudinal image of ultrasonography (with linear transducer) showed similar findings in the left lobe of the thyroid with linear echogenic strands (dotted arrow); normal thyroid tissues could also be seen (thin arrow), and the capsule of the thyroid was invaded (thick arrow); (C) longitudinal image of ultrasonography (with linear transducer) showed pathologically confirmed involved lymph nodes in the right cervical III, IV and VI regions appeared as markedly hypoechoic lymph nodes lacking echogenic hilum. DLBCL, diffuse large B-cell lymphoma; PTL, primary thyroid lymphoma.

which might be due to more extensive local invasion in ATC compared to PTL.

In our study, most ATC and PTL appeared as large, solid, and ill-defined masses. However, there is statistic significant difference in some sonographic features, including sonographic pattern, echogenic strands, markedly hypoechoic, calcification and enhanced posterior echo.

A total of $25.9 \%(7 / 27)$ of PTLs presented with diffuse type, occupying entire thyroid without normal gland or most of the thyroid gland. Similar to the findings of the previous studies $(20,26)$, all PTLs in our study are of B-cell lymphoma and predominantly of diffuse large-cell type, and the second most common histological type is MALT lymphoma. Most DLBCLs in our study showed nodular type (10/15, 66.7\%). Compared to PTLs, all ATC cases were nodular type and limited to one lobe of thyroid, suggesting that PTL showed a propensity for more extensive disease at initial diagnosis. Plenty of ATC and PTL in our study showed direct invasion to the surrounding structures. Relevant literatures revealed that patients with ATC present with extensive local invasion at initial disease presentation in $15 \%$ to $50 \%$ of patients $(27,28)$, and some research reported that invasion to the surrounding structures is rare in PTL $(29,30)$. However, a few studies also reported PTL with aggressive appearance $(31,32)$. Thus, the ultrasonographic features of local invasion were not specific enough to differentiate ATC from PTL.

Another typical sonographic feature for PTL is linear echogenic strands. In our study, most of PTLs presented with echogenic strands $(25 / 27,92.6 \%)$, and only one lesion $(1 / 15,6.7 \%)$ of ATC presented with echogenic strands. Some studies suggested that sonographic findings of PTL were correlated with pathologic findings. Orita et al. (33) and Nam et al. (29) indicated that the hypoechoic areas of PTL were consistent with lymphoepithelial lesions containing neoplastic cells, whereas echogenic structures within or around the lesions were consistent with fibrotic tissue and normal thyroid tissue. Ota (9) reported a destructive linear pattern which might suggest the transformation of MALT lymphoma into a high-grade malignant lymphoma. In their study, all of the 15 MALT lymphoma of the head and neck presented with echogenic structures whereas two DLBCLs of thyroid showed linear echogenic strands and one DLBCL of parotid gland showed destructive linear echogenic strands, and the remaining 13 DLBCLs showed no echogenic strands. However, in our study, all (8/8) MALTs and 73.3\% (11/15) of DLBCLs had linear echogenic strands, and 2 DLBCLs had destructive linear strands.

With the image of ultrasound, it is hard to identify the pathological type of PTL. Pathology and immunohistochemistry are the main method of clinical diagnosis of PTL and guiding 

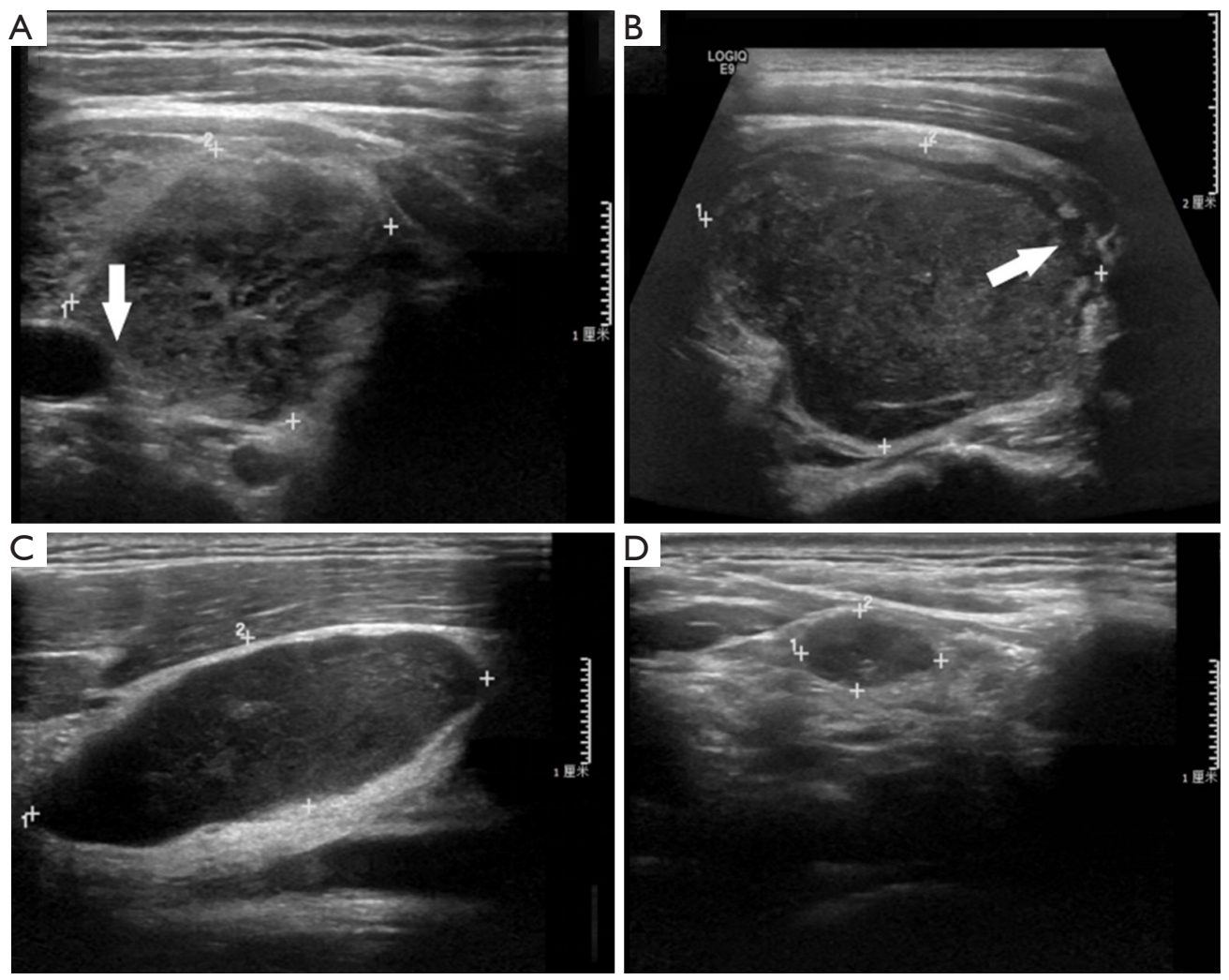

Figure 3 Ultrasonography of diffuse large B-cell lymphoma of the thyroid in a 46-year-old man presented with rapidly growing neck swelling for 10 days. (A) Transverse image of ultrasonography (with linear transducer) showed nodular type of PTL. There was an irregularshaped hypoechoic mass in the right lobe of the thyroid. The lesion had invaded the vascular wall (thick arrow); (B) transverse image of ultrasonography (with linear transducer) showed a markedly hypoechoic mass in the left lobe of the thyroid with invasion of thyroid capsule (thick arrow). We could also see echo enhancement behind the lesion; (C,D) longitudinal image of ultrasonography (with linear transducer) showed pathologically confirmed involved lymph nodes in the left cervical III region appeared as markedly hypoechoic lymph nodes lacking echogenic hilum.

therapy (24). Except the difference in cell morphology, cytokine receptor is also an important way of identifying cell types, and it is also becoming a hotspot of current research. For example, CD3 is always expressed in T-cell lymphoma and B-cell lymphoma are commonly found CD20, CD22 and CD79a $(34,35)$. In addition, analysis of ubiquitous lymphoma cells sequences is also a new technology to distinguish different types of PTL (36).

In our ATC group, 52.9\% (9/17) of ATC cases showed intratumoral calcification, including microcalcification, macrocalcification and mixed calcification. In previous studies by Takashima et al. (30) and Ishikawa et al. (31), the authors demonstrated calcification was one of the dominant computed tomographic findings in ATC. Lee et al. (32) also reported that $44.4 \%$ of anaplastic carcinomas showed nodular calcification. In contrast, none of the PTL cases showed intratumoral calcification.

Markedly hypoechogenicity is one of typical features of PTL $(10,37)$, however, previous studies had inconsistent results for echogenicity in ATC. Suh et al. (38) reported that markedly hypoechogenicity is a common US feature in ATC $(52.9 \%, 9 / 17)$, and Hahn et al. (5) reported hypoechogenicity is more common in ATC $(61.5 \%, 16 / 26)$. In our study, marked hypoechogenicity occurred more frequently in PTL than ATC with significantly difference, but this result still need further investigations.

Enhanced posterior echo has also been considered to be the specific feature of all types of PTL (9). In the present study, enhanced posterior echoes are observed in 23 of 27 PTL cases (85.2\%) whereas only 5 of 15 (33.3\%) ATC cases showed this feature. An enhanced posterior echo in PTL is possibly due to densely and uniformly 

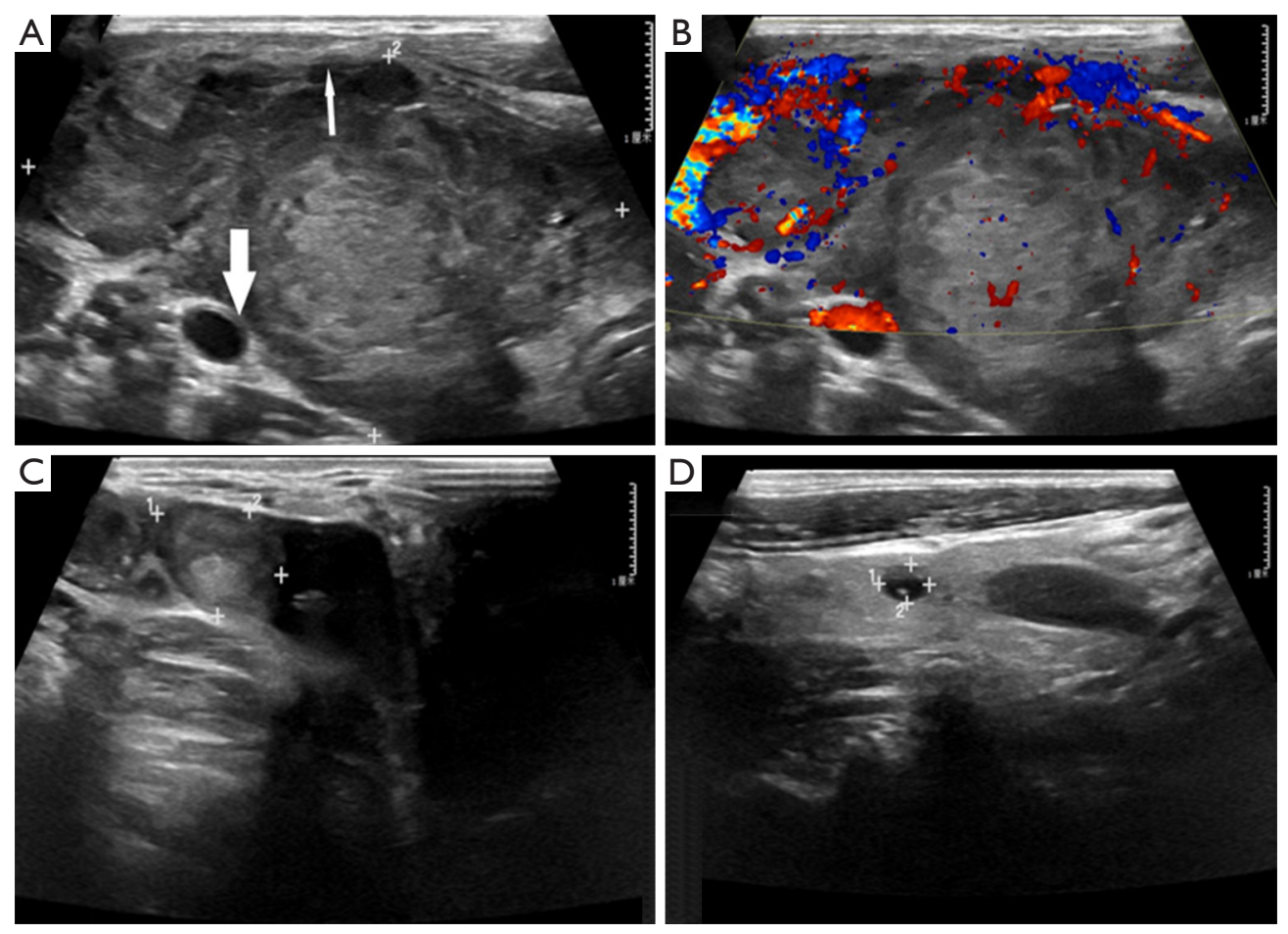

Figure 4 Ultrasonography of ATC in a 62-year-old man presented with rapidly neck swelling in 2 months. (A) Transverse image of ultrasonography (with linear transducer) of the right lobe of thyroid showed an irregular shaped, ill-defined, heterogeneous hypoechoic mass with invasion of thyroid capsule and surrounding soft tissues (thin arrow). The boundary between the lesion and vascular wall was vague, which was proved by pathology as vascular wall invasion (thick arrow); (B) Color Doppler imaging showed a rich internal and peripheral blood flow pattern; (C) transverse image of ultrasonography (with linear transducer) showed enlarged lymph node pathologically confirmed as lymph nodes metastasis; (D) transverse image of ultrasonography (with linear transducer) of a well-defined anechoic nodule in the left lobe of thyroid, which was proved by pathology as nodular goiter. ATC, anaplastic thyroid carcinoma.
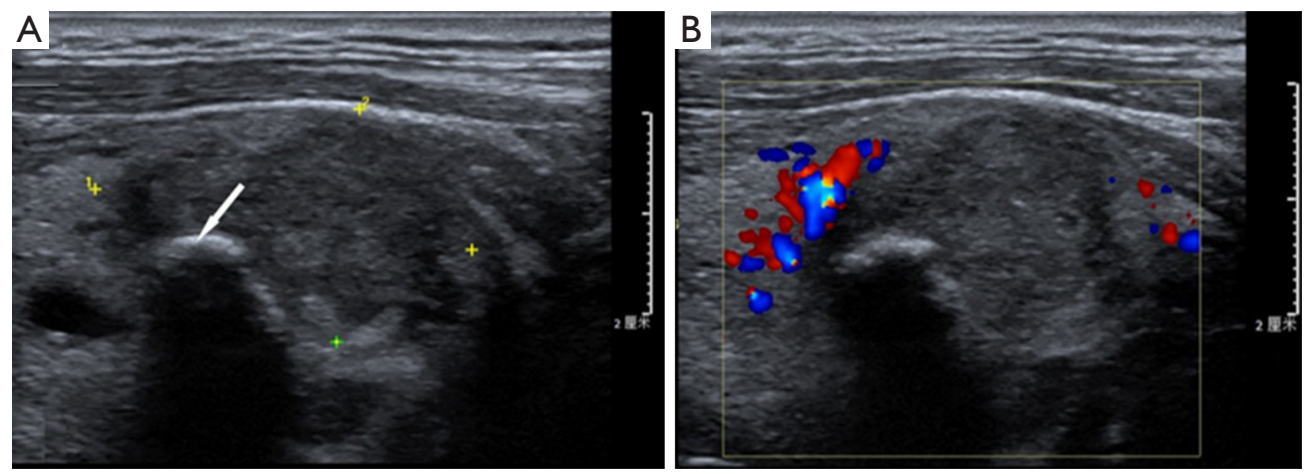

Figure 5 Ultrasonography of ATC in a 64-year-old man presented with hoarseness for 10 days. (A) Longitudinal image of ultrasonography (with linear transducer) of ATC in the right lobe of thyroid showed an irregular shaped, ill-defined, heterogeneous hypoechoic mass with cambered macrocalcification (arrow); (B) Color Doppler imaging showed a rich peripheral blood flow. 
proliferated lymphoma cells in the lesion, in contrast, ATC is characterized by pleomorphic cells with a spectrum of morphologies (spindle, giant cell, rhabdoid and squamoid). Most ATC lesions are composed of undifferentiated tumor cells and well-differentiated thyroid carcinoma which may have a dense and compact cellular structure (39-41). Meanwhile, the microcalcifications are the other reason leading to acoustic attenuation behind the lesions of ATC.

Our results also showed that the patients with PTL more often have a background of Hashimoto's thyroiditis $(59.3 \%)$ than that of ATC (20\%). It has been reported that preexisting Hashimoto's thyroiditis is a wellrecognized risk factor for the development of PTL, and the average incidence of Hashimoto's thyroiditis in patients with PTL is $80 \%$, ranging from $30-100 \%$ in previous studies $(12,14)$. There is also evidence that large cell lymphoma probably evolves from persistent low-grade MALT malignant lymphoma, suggesting a morphological progression from chronic lymphocytic thyroiditis to lowgrade MALT lymphoma, and subsequently, to highgrade large-cell lymphoma (42). So, we think that the high incidence of associated Hashimoto's thyroiditis may facilitate the differentiation of malignant lymphoma from undifferentiated carcinoma.

The coexistence of well-differentiated and ATCs has been well demonstrated in several studies (43-46), however, whether it arises from a preexisting well-differentiated thyroid carcinoma (e.g., papillary thyroid carcinoma, PTC) is not clear. That might explain why ATC present with some characteristics of PTC, such as microcalcifications. In our study, 4/15 patient had zones of PTC $(n=3)$ or adenocarcinoma $(\mathrm{n}=1)$ in the ATC lesion, and 1 patient had PTC in the contralateral lobe of thyroid. One patient with ATC had a surgery of local excision of one side lobe because of PTC 1 year ago.

Defining a differential diagnosis of PTL and ATC is usually a challenging clinical issue. To our knowledge, this study was the first to compare the sonographic findings between PTL and ATC. In our study, PTL presented with diffuse or mixed type can be differentiate from ATC more easily; however, differentiation between PTL presented with nodular type and ATCs is not easily established on imaging. Some sonographic features such as a markedly hypoechogenicity, the presence of an enhanced posterior echo and echogenic strands, lack of calcification and associated Hashimoto's thyroiditis were valuable for distinguishing PTL from ATC. In contrast, lesion size, echo texture, margin, shape, cystic change, vascular pattern and local invasion were not specific features for differential diagnosis.

There are some limitations to this study. First, overall survival of patients has not been record, so we cannot compare prognosis of the two diseases. Second, we have a small population size of PTL and ATC because both PTL and ATC are rare diseases, and it is difficult to obtain a large integrated number of cases for analysis.

\section{Acknowledgements}

Funding: This work was supported by Beijing Hope Run Special Fund of China Cancer Research Foundation (CCRF) (No. LC2016A04); Peking Union Medical College (PUMC) Youth Fund and the Fundamental Research Funds for the Central Universities (No. 2017320015).

\section{Footnote}

Conflicts of Interest: The authors have no conflicts of interest to declare.

Ethical Statement: The study was approved by the Hospital Ethics Committee (No. NCC2016YZ-15). Each patient was consent informed.

\section{References}

1. Smallridge RC, Copland JA. Anaplastic thyroid carcinoma: pathogenesis and emerging therapies. Clin Oncol (R Coll Radiol) 2010;22:486-97.

2. Nachalon Y, Stern-Shavit S, Bachar G, et al. Aggressive Palliation and Survival in Anaplastic Thyroid Carcinoma. JAMA Otolaryngol Head Neck Surg 2015;141:1128-32.

3. Hirokawa M, Sugitani I, Kakudo K, et al. Histopathological analysis of anaplastic thyroid carcinoma cases with long-term survival: A report from the Anaplastic Thyroid Carcinoma Research Consortium of Japan. Endocr J 2016;63:441-7.

4. Are C, Shaha AR. Anaplastic thyroid carcinoma: biology, pathogenesis, prognostic factors, and treatment approaches. Ann Surg Oncol 2006;13:453-64.

5. Hahn SY, Shin JH. Description and Comparison of the Sonographic Characteristics of Poorly Differentiated Thyroid Carcinoma and Anaplastic Thyroid Carcinoma. J Ultrasound Med 2016;35:1873-9.

6. Brignardello E, Palestini N, Felicetti F, et al. Early surgery 
and survival of patients with anaplastic thyroid carcinoma: analysis of a case series referred to a single institution between 1999 and 2012. Thyroid 2014;24:1600-6.

7. Sakorafas GH, Kokkoris P, Farley DR. Primary thyroid lymphoma (correction of lympoma): diagnostic and therapeutic dilemmas. Surg Oncol 2010;19:e124-9.

8. Mishra P, Banerjee D, Gujral S. T-cell Lymphoma of Thyroid Gland with Lennert Type of Morphology: A Case Report and Review of the Literature. Head Neck Pathol 2016;10:321-6.

9. Ota H, Ito Y, Matsuzuka F, et al. Usefulness of ultrasonography for diagnosis of malignant lymphoma of the thyroid. Thyroid 2006;16:983-7.

10. Xia Y, Wang L, Jiang Y, et al. Sonographic appearance of primary thyroid lymphoma-preliminary experience. PLoS One 2014;9:e114080.

11. Danialan R, Tetzlaff MT, Torres-Cabala CA, et al. Cutaneous metastasis from anaplastic thyroid carcinoma exhibiting exclusively a spindle cell morphology. A case report and review of literature. J Cutan Pathol 2016;43:252-7.

12. Onoda N, Sugitani I, Higashiyama T, et al. Concept and design of a nationwide prospective feasibility/ efficacy/safety study of weekly paclitaxel for patients with pathologically confirmed anaplastic thyroid cancer (ATCCJ-PTX-P2). BMC Cancer 2015;15:475.

13. Matsuzuka F, Miyauchi A, Katayama S, et al. Clinical aspects of primary thyroid lymphoma: diagnosis and treatment based on our experience of 119 cases. Thyroid 1993;3:93-9.

14. Antonelli A, Miccoli P, Fallahi P, et al. Role of neck ultrasonography in the follow-up of children operated on for thyroid papillary cancer. Thyroid 2003;13:479-84.

15. Sarinah B, Hisham AN. Primary lymphoma of the thyroid: diagnostic and therapeutic considerations. Asian J Surg 2010;33:20-4.

16. Brown RE, Harave S. Diagnostic imaging of benign and malignant neck masses in children-a pictorial review. Quant Imaging Med Surg 2016;6:591-604.

17. Yuan J, Lo G, King AD. Functional magnetic resonance imaging techniques and their development for radiation therapy planning and monitoring in the head and neck cancers. Quant Imaging Med Surg 2016;6:430-48.

18. Cha C, Chen H, Westra WH, et al. Primary thyroid lymphoma: can the diagnosis be made solely by fine-needle aspiration? Ann Surg Oncol 2002;9:298-302.

19. Hwang YC, Kim TY, Kim WB, et al. Clinical characteristics of primary thyroid lymphoma in Koreans.
Endocr J 2009;56:399-405.

20. Derringer GA, Thompson LD, Frommelt RA, et al. Malignant lymphoma of the thyroid gland: a clinicopathologic study of 108 cases. Am J Surg Pathol 2000;24:623-39.

21. Ha CS, Shadle KM, Medeiros LJ, et al. Localized nonHodgkin lymphoma involving the thyroid gland. Cancer 2001;91:629-35.

22. Skarsgard ED, Connors JM, Robins RE. A current analysis of primary lymphoma of the thyroid. Arch Surg 1991;126:1199-203; discussion 203-4.

23. Us-Krasovec M, Golouh R, Auersperg M, et al. Anaplastic thyroid carcinoma in fine needle aspirates. Acta Cytol 1996;40:953-8.

24. Ha EJ, Baek JH, Lee JH, et al. Core needle biopsy could reduce diagnostic surgery in patients with anaplastic thyroid cancer or thyroid lymphoma. Eur Radiol 2016;26:1031-6.

25. Sasai K, Yamabe H, Haga H, et al. Non-Hodgkin's lymphoma of the thyroid. A clinical study of twenty-two cases. Acta Oncol 1996;35:457-62.

26. Thieblemont C, Mayer A, Dumontet C, et al. Primary thyroid lymphoma is a heterogeneous disease. J Clin Endocrinol Metab 2002;87:105-11.

27. Thompson LD, Wieneke JA, Paal E, et al. A clinicopathologic study of minimally invasive follicular carcinoma of the thyroid gland with a review of the English literature. Cancer 2001;91:505-24.

28. Kim EE. Thyroid Cancer: A Comprehensive Guide to Clinical Management. J Nucl Med 2017;58:1013.

29. Nam M, Shin JH, Han BK, et al. Thyroid lymphoma: correlation of radiologic and pathologic features. J Ultrasound Med 2012;31:589-94.

30. Takashima S, Morimoto S, Ikezoe J, et al. Primary thyroid lymphoma: comparison of CT and US assessment. Radiology 1989;171:439-43.

31. Ishikawa H, Tamaki Y, Takahashi M, et al. Comparison of primary thyroid lymphoma with anaplastic thyroid carcinoma on computed tomographic imaging. Radiat Med 2002;20:9-15.

32. Lee JW, Yoon DY, Choi CS, et al. Anaplastic thyroid carcinoma: computed tomographic differentiation from other thyroid masses. Acta Radiol 2008;49:321-7.

33. Orita Y, Sato Y, Kimura N, et al. Characteristic ultrasound features of mucosa-associated lymphoid tissue lymphoma of the salivary and thyroid gland. Acta Otolaryngol 2014;134:93-9.

34. Li L, Guo X, Shi X, et al. Ionic CD3-Lck interaction 
regulates the initiation of T-cell receptor signaling. Proc Natl Acad Sci U S A 2017;114:E5891-9.

35. Wang JH, Chen L, Ren K. Identification of primary thyroid lymphoma with medical imaging: A case report and review of the literature. Oncol Lett 2014;8:2505-8.

36. Shi L, Zhang Y, Feng L, et al. Multi-omics study revealing the complexity and spatial heterogeneity of tumorinfiltrating lymphocytes in primary liver carcinoma. Oncotarget 2017;8:34844-57.

37. Kim EH, Kim JY, Kim TJ. Aggressive primary thyroid lymphoma: imaging features of two elderly patients. Ultrasonography 2014;33:298-302.

38. Suh HJ, Moon HJ, KwakJY, et al. Anaplastic thyroid cancer: ultrasonographic findings and the role of ultrasonography-guided fine needle aspiration biopsy. Yonsei Med J 2013;54:1400-6.

39. Suzuki S, Fujisaki S, Takashina M, et al. A case of anaplastic thyroid carcinoma with surgical treatment. Gan To Kagaku Ryoho 2014;41:2512-4.

40. Molinaro E, Romei C, Biagini A, et al. Anaplastic thyroid carcinoma: from clinicopathology to genetics and advanced therapies. Nat Rev Endocrinol 2017. [Epub ahead of print]. 41. Smallridge RC, Ain KB, Asa SL, et al. American Thyroid
Association guidelines for management of patients with anaplastic thyroid cancer. Thyroid 2012;22:1104-39.

42. Yokoyama J, Ito S, Ohba S, et al. Problems of primary T-cell lymphoma of the thyroid gland--a case report. World J Surg Oncol 2012;10:58.

43. Costaldi ME, Zhou Z. Case report: different metastatic components from anaplastic thyroid carcinoma in mediastinal and neck lymph nodes simultaneously diagnosed by FNA. Diagn Cytopathol 2014;42:694-9.

44. Oishi N, Kondo T, Ebina A, et al. Molecular alterations of coexisting thyroid papillary carcinoma and anaplastic carcinoma: identification of TERT mutation as an independent risk factor for transformation. Mod Pathol 2017. [Epub ahead of print].

45. Ambelil M, Sultana S, Roy S, et al. Anaplastic Transformation in Mandibular Metastases of Follicular Variant of Papillary Thyroid Carcinoma: A Case Report and Review of the Literature. Ann Clin Lab Sci 2016;46:552-6.

46. Xu B, Ghossein R. Genomic Landscape of poorly Differentiated and Anaplastic Thyroid Carcinoma. Endocr Pathol 2016;27:205-12.
Cite this article as: Gu LS, Cui NY, Wang Y, Che SN, Zou SM, He W, Liu JY, Gong XT. Comparison of sonographic characteristics of primary thyroid lymphoma and anaplastic thyroid carcinoma. J Thorac Dis 2017;9(11):4774-4784. doi: $10.21037 /$ jtd. 2017.09 .48 